\title{
ON DISCRETE SAMPLING OF TIME-VARYING CONTINUOUS-TIME SYSTEMS
}

\author{
P.M. Robinson*
}

Discussion paper

No. EM/2007/520

June 2007
The Suntory Centre

Suntory and Toyota International Centres for Economics and Related Disciplines

London School of Economics and Political Science Houghton Street

London WC2A $2 A E$

Tel: 02079556679

\footnotetext{
* Address: Department of Economics, London School of Economics, Houghton Street, London WC2A 2AE, United Kingdom. Tel: 44-020-7955-7516, Fax: 44-020-7955-6592,

email: p.m.robinson@lse.ac.uk.

Research supported by ESRC Grant RES-062-23-0036.
} 


\begin{abstract}
We consider a multivariate continuous time process, generated by a system of linear stochastic differential equations, driven by white noise and involving coefficients that possibly vary over time. The process is observable only at discrete, but not necessarily equally-spaced, time points (though equal spacing significantly simplifies matters). Such settings represent partial extensions of ones studied extensively by A.R. Bergstrom. A model for the observed time series is deduced. Initially we focus on a first-order model, but higher-order ones are discussed in case of equally-spaced observations. Some discussion of issues of statistical inference is included.
\end{abstract}

Key Words and Phrases: Stochastic differential equations; time-varying coefficients; discrete sampling; irregular sampling.

JEL Classification: C32

(C) The author. All rights reserved. Short sections of text, not to exceed two paragraphs, may be quoted without explicit permission provided that full credit, including $₫$ notice, is given to the source. 


\section{INTRODUCTION}

Continuous-time modelling in econometrics was pioneered by Rex Bergstrom. At least if calendar effects can be ignored, econometric time series data are usually observed, perhaps after temporal aggregation, at discrete, regular intervals of time. On the other hand, the interval of measurement may have no economic significance, in which case an underlying continuous-time process can be imagined. If so, modelling based on the continuous-time process may be more natural and elegant, than modelling directly the discrete-time observations. In his 1966 Econometrica paper and 1967 book, Bergstrom $(1966,1967)$ initiated continuous-time modelling of discrete-time macroeconometric data as a topic of econometric research. Consequently, interest expanded in the 1970's, as illustrated by the collection of articles by a number of researchers that Bergstrom (1976) edited. On a personal note, I was then a young researcher whose interest in the topic was considerably stimulated by reading Rex's early work. He kindly published a chapter of my PhD thesis in Bergstrom (1976), and was always immensely kind and helpful to me in my career.

Building on his early development of the subject, Rex Bergstrom continued to contribute to it very significantly and uniquely for the rest of his life. His work included several advances in econometric theory, developing exact and approximate discrete time models and justifying rules of large sample statistical inference on parameters in 
the underlying continuous-time model (e.g. Bergstrom, 1983); this work was marked by considerable rigour and care, and matched the challenge of the topic. Perhaps even more distinctively, he successfully built and empirically estimated multiple equation models of the economy (e.g. Bergstrom, 1984); this work was considerably informed by economic theory, and his approach has been very influential, having been adopted by a number of empirical macroeconometric researchers internationally. Remarkably, he continued to be productive long into retirement, as illustrated by his final, posthumously-published book, Bergstrom and Nowman (2007).

Developing statistical inference on continuous-time models using discrete-time data is an ambitious undertaking, as Rex Bergstrom was well aware. Intuitively, this is obvious from the fact that any sequence of discrete observations can be interpolated by uncountably many continuous paths. This leads to the classical "aliasing" phenomenon (most frequently discussed in the context of stationary processes, where the spectral density of the equally-spaced discrete sequence is generated from that of the continuous one by the "folding formula"), and thence to a lack of identifiability. The folding formula produces an upper bound for the continuous-time spectral density (because it is everywhere non-negative), but uncountably many versions of it are consistent with a given discrete-time spectral density.

However, a parsimonious parametric model for macroeconomic processes is usually necessary due to shortage of data, especially in a multivariate setting, and such 
a model can considerably alleviate the identification problem. Typically, the model has been a system of constant-coefficientstochastic differential equations. Then it is possible (see Phillips, 1973) that the parameters in the model can be at least locally identified, though this can be difficult to demonstrate analytically. Unfortunately, though an exact discrete-time model can be written down, and may belong to the familiar autoregressive moving average (ARMA) class, the parameters of the discretetime model can be extremely complicated functions of the underlying parameters; their analytic form depends, indeed, on whether any roots of the continuous-time autoregressive operator are repeated, and if so, on their multiplicities. Consequently, interest in simpler, discrete-time "approximations" developed, for example ones generated by replacing derivatives by differences (see e.g.Bergstrom, 1966). However, there is an element of arbitrariness in the choice of approximation, and for a given model a continuum of "discrete approximations" can be justified, utilization of each leading to different parameter estimates and possibly different conclusions about economic behaviour (see Robinson, 1977a). One solution is to employ the basic discrete ARMA structure that is derived, and estimate this unconstrainedly, ignoring the restrictions. However, the ARMA typically has more coefficients than the initial continuous-time model had, so inefficiency results. As a further pitfall, the linear stochastic differential equations are typically assumed to be driven by white noise, or more generally, in the stationary case, the process has a rational spectral density. This assumption 
cannot be taken for granted, hence, specifying the dynamics of the unobservable input process is a hazardous undertaking, and misspecification leads to a misspecified "exact" discrete model.

Nevertheless, a considerable body of work has developed, in large part due to Rex Bergstrom and his students, treating the modelling of systems with or without predetermined variables, developing the linear model to allow for forms of nonlinearity, and extending stationary models to nonstationary ones, to reflect the growing econometric interest in nonlinear and nonstationary dynamics in purely discrete time. The literature is described in the historical review article Bergstrom (1988). In fact, this literature, beginning over 40 years ago, with its focus on macroeconomic time series, is reflected in considerable recent activity with continuous-time modelling in finance. Here the main difficulties facing Bergstrom, discussed above, are reduced due to the very finely spaced sampling of many financial time series, and their considerable length. Nevertheless, high frequency financial data and microstructure noise affect model formulations in a way that introduces considerable analytic complexity and calls for new inferential approaches. Phillips and Yu (2005a,b, 2006) emphasize the magnitude of finite sample bias due to the discrete sampling, and its economic implications.

The bulk of research on continuous-time modelling has assumed constancy of parameters across time. On the other hand, time-varying models have over the years 
become a focus of study within mainstream discrete time series econometrics and statistics. In large part this is due to a need to describe one or more changes in structure, due to economic, political or natural events that have a sudden effect on the economy. There has also been considerable work with parameters that can change over time, possibly smoothly and possibly with respect to each observation point, or perhaps with discontinuous jumps. Here we will discuss an extension of a famous result for time invariant continuous-time models that was alluded to above: a discrete-time equally-spaced sequence from a continuous time ARMA process has a certain discrete ARMA representation, as found by Bartlett (1946) and subsequent researchers. We attempt to extend this sort of result to a setting that allows general variation across time in coefficients. We also allow for multivariate data, and additionally consider the possibility of irregularly-spaced sampling. Finally we discuss issues of modelling statistical inference.

\section{TIME-VARYING CONTINUOUS-TIME MODEL AND DISCRETE SAMPLING}

On the real interval $T=\left[t_{0},.\right)$, define $X(t), t \in T$, to be a mean-zero, real-valued, $p$-dimensional vector homogeneous random process with orthogonal increments, that 
is

$$
\begin{array}{rlrl}
E\left(X\left(t_{1}\right)-X\left(t_{2}\right)\right)\left(X\left(t_{3}\right)-X\left(t_{4}\right)\right)^{\prime} & =\left(t_{1}-t_{2}\right) I_{p}, & t_{1}=t_{3}>t_{2}=t_{4} \\
& =0, & & t_{1}>t_{2} \geq t_{3}>t_{4},
\end{array}
$$

$I_{p}$ being the $p$-rowed unit matrix and the prime denoting transposition.

Initially, let $A(t)$ and $B(t)$ be respectively $q \times q$ and $q \times p$ matrices of real-valued, bounded, measurable functions and consider the system

$$
\begin{aligned}
d Y(t) & =A(t) Y(t) d t+B(t) d X(t), \quad t \in T ; \\
Y\left(t_{0}\right) & =Y_{0} .
\end{aligned}
$$

Many processes $Y(t)$ that arise, not only in economics but also the natural sciences and engineering, are so modelled, but often with $A(t)$ and $B(t)$ assumed constant across $t$, as in Rex Bergstrom's work. Note that changing $A(t)$ with $t$ entails timevarying dynamics, whereas changing $B(t)$ with $t$ entails time-varying conditional heteroscedasticity.

It is assumed that observations can be made only at countably many points in $T$, $t_{1}<t_{2}<\cdots$. Indeed the property (1) may limit the accuracy with which $Y(t)$ can be continuously recorded. We deduce a model for $Y\left(t_{j}\right), j \geq 1$.

Let there exist, for all $t$, a nonsingular fundamental matrix $\Phi(t)$ for $A(t)$ (Cod- 
dington and Levinson, 1955, pp.69-71), satisfying

$$
\begin{aligned}
\frac{d}{d t} \Phi(t) & =-\Phi(t) A(t), \quad t>t_{0} \\
\Phi\left(t_{0}\right) & =I_{q}
\end{aligned}
$$

Suppose, in addition to (1), that

$$
\begin{aligned}
E Y_{0} & =0 \\
E Y_{0} Y_{0}^{\prime} & =\Omega<\infty \\
E Y_{0}\left(X\left(t_{2}\right)-X\left(t_{1}\right)\right)^{\prime} & =0, \quad t_{0}<t_{1} \leq t_{2} .
\end{aligned}
$$

A solution will be described as unique if it is unique in the mean square sense considered by Friedman (1975, Theorem 1.1). The following theorem demonstrates equivalence in second-order moment properties of continuous-time and discrete-time solutions.

Theorem: Let $Y_{c}(t), t \in T$, be the unique solution of (2). For any given sequence $t_{1}, t_{2}, . . \in T$, where $t_{0}<t_{1}<t_{2} \cdots$, let $Y_{d}\left(t_{j}\right), j \geq 1$, be the unique solution of

$$
\begin{aligned}
Y\left(t_{j}\right)-\Phi\left(t_{j}\right)^{-1} \Phi\left(t_{j-1}\right) Y\left(t_{j-1}\right) & =Z\left(t_{j}\right), \quad j \geq 1 \\
Y\left(t_{0}\right) & =Y_{0}
\end{aligned}
$$


where

$$
\begin{aligned}
E Z\left(t_{j}\right) & =0, \\
E Z\left(t_{j}\right) Z\left(t_{j}\right)^{\prime} & =\Phi\left(t_{j}\right)^{-1} \Sigma_{j} \Phi\left(t_{j}\right)^{-1 \prime}, \\
E Z\left(t_{j}\right) Z\left(t_{k}\right)^{\prime} & =0, \quad j \neq k, \\
\Sigma_{j} & =\int_{t_{j-1}}^{t_{j}} \Phi(t) B(t) B(t)^{\prime} \Phi(t)^{\prime} d t .
\end{aligned}
$$

Then

$$
E Y_{c}\left(t_{j}\right) Y_{c}\left(t_{k}\right)^{\prime}=E Y_{d}\left(t_{j}\right) Y_{d}\left(t_{k}\right)^{\prime},
$$

for all $j, k$.

Proof. Premultiplication of (2) by $\Phi(t)$ and use of (3) produces

$$
d(\Phi(t) Y(t))=\Phi(t) B(t) d X(t)
$$

whence integration over $\left(t_{j-1}, t_{j}\right), j \geq 1$, produces

$$
\Phi\left(t_{j}\right) Y\left(t_{j}\right)-\Phi\left(t_{j-1}\right) Y\left(t_{j-1}\right)=\int_{t_{j-1}}^{t_{j}} \Phi(t) B(t) d X(t),
$$

which may be written as (5), with

$$
Z\left(t_{j}\right)=\Phi\left(t_{j}\right)^{-1} \int_{t_{j-1}}^{t_{j}} \Phi(t) B(t) d X(t) .
$$

It is readily verified that (1) implies (6). Now (5) has the unique solution

$$
Y_{d}\left(t_{j}\right)=\Phi\left(t_{j}\right)^{-1}\left(Y_{0}+\sum_{i=1}^{j} \Phi\left(t_{i}\right) Z\left(t_{i}\right)\right), \quad j \geq 1,
$$


so that, from (4), (6)

$$
E Y_{d}\left(t_{j}\right) Y_{d}\left(t_{k}\right)^{\prime}=\Phi\left(t_{j}\right)^{-1}\left(\Omega+\Sigma_{1}+\cdots+\Sigma_{\min (j, k)}\right) \Phi\left(t_{k}\right)^{-1 \prime} .
$$

On the other hand, from Soong (1973, p.154) a solution of (2) is

$$
Y_{c}(t)=\Phi(t)^{-1}\left(Y_{0}+\int_{t_{0}}^{t} \Phi(u) B(u) d X(u)\right),
$$

and this is unique (Friedman, 1975, Theorem 1.1). Thus

$$
\begin{aligned}
E Y_{c}\left(t_{j}\right) Y_{c}\left(t_{k}\right)^{\prime} & =\Phi\left(t_{j}\right)^{-1}\left(\Omega+\int_{0}^{\min \left(t_{j}, t_{k}\right)} \Phi(u) B(u) B(u)^{\prime} \Phi(u)^{\prime} d u\right) \Phi\left(t_{k}\right)^{-1^{\prime}} \\
& =E Y_{d}\left(t_{j}\right) Y_{d}\left(t_{k}\right)^{\prime} .
\end{aligned}
$$

This completes the proof.

This "skip -sampling" result could be extended to cover temporally-aggregated data. It would be more difficult to deal with a situation in which different elements of $Y(t)$ are observed at different time points.

In the usual constant-coefficient case, $A(t) \equiv A$, we have, with $t_{0}=0, \Phi(t)=e^{-A t}$, where for a square matrix $Z, e^{Z}=1+\sum_{j=1}^{\infty} Z^{j} / j$ ! when the series converges. Then we deduce that the AR coefficient matrix $\Phi\left(t_{j}\right)^{-1} \Phi\left(t_{j-1}\right)=e^{A\left(t_{j}-t_{j-1}\right)}$. In the regularlyspaced case where $t_{j}-t_{j-1}=\Delta$, the latter matrix becomes $e^{A \Delta}$, as in Rex Bergstrom's work. With irregular spacing we have a multivariate extension of a result obtained by Robinson (1977b). 
More generally, for a scalar integrable function $f(t)$, suppose $A(t)=A f(t)$, where $\Phi(t)=e^{-A g(t)}$ with $g(t)=\int_{-\infty}^{t} f(u) d u$. Thus $\Phi\left(t_{j}\right)^{-1} \Phi\left(t_{j-1}\right)=e^{A\left\{g\left(t_{j}\right)-g\left(t_{j-1}\right)\right\}}$, in which we may write $g\left(t_{j}\right)-g\left(t_{j-1}\right)=\int_{t_{j-1}}^{t_{j}} f(u) d u$. For example, in the periodic case $f(t)=\cos (b t), b \neq 0$, we have $g(t)=-\sin (b t) / b$ and thence $\Phi\left(t_{j}\right)^{-1} \Phi\left(t_{j-1}\right)=$ $e^{A\left\{\sin \left(b t_{j}\right)-\sin \left(b t_{j-1}\right)\right\} / b}=e^{2 A \sin \left\{b\left(t_{j}-t_{j-1}\right) / 2\right\} \cos \left\{b\left(t_{j}+t_{j-1}\right) / 2\right\}}$. With regular spacing, $t_{j+1}-$ $t_{j} \equiv \Delta$, this becomes $e^{2 A \sin (b \Delta / 2) \cos (b(t-\Delta / 2))}$ at $t_{j}=t$. Thus the discrete-time model also has a periodic structure.

We have focussed on closed systems, containing no predetermined or exogenous variables. An open system, which contains a vector $U(t)$ of such observable variables is

$$
d Y(t)=A(t) Y(t) d t+C(t) U(t)+B(t) d X(t), \quad t \in T .
$$

We can infer from the Theorem proof, by simply substituting $C(t) U(t)+B(t) d X(t)$ for $B(t) d X(t)$, a model that includes $Y(t)$ only discretely, but depends on the continuous $U(t)$, as well as the continuous $X(t)$. However, it is likely that $U(t)$ will only be observed discretely. This mirrors the problem encountered with constant-coefficient models, where a full discrete model has been deduced by either "exactly", by an implicit assumption about the continuous time dynamics of $U(t)$, which is inevitably a source of misspecification if incurred, or a discrete "approximation", which again really has the staus of a good approximation for suitable continuous time dynamics (see e.g. Phillips, 1974, Sargan, 1974, Robinson, 1976, Bergstrom, 1983). Of course 
an allowance for irregular spacing raises the possibility that $U(t)$ could be observed at different times from $Y(t)$, which would make for further difficulties.

\section{Higher-Order Extension}

Successive application of the step from (1) to (5) yields a sampled version of the $r^{\text {th }}$-order vector continuous-time model

$$
\begin{aligned}
d D^{r-1} Y(t) & =\sum_{i=1}^{r} A_{i}(t) D^{i-1} Y(t) d t+B(t) d X(t) \\
Y\left(t_{0}\right) & =Y_{0}
\end{aligned}
$$

where $D^{j}=d^{j} / d t^{j}$. First write (7) in the form (2) with $X(t), Y(t), A(t), B(t)$ there replaced by

$$
\left[\begin{array}{c}
0 \\
0 \\
\vdots \\
0 \\
X(t)
\end{array}\right],\left[\begin{array}{c}
Y(t) \\
D Y(t) \\
\vdots \\
D^{r-2} Y(t) \\
D^{r-1} Y(t)
\end{array}\right],\left[\begin{array}{ccc}
0 & I_{q} & 0 \\
0 & \ddots & \\
\vdots & & I_{q} \\
A_{1}(t) & & A_{2}(t) \cdots A_{r}(t)
\end{array}\right],\left[\begin{array}{cc}
\cdots & 0 \\
\cdots & 0 \\
\cdots \\
\cdots \\
\cdots \\
\cdots
\end{array}\right]
$$

respectively.

For simplicity consider only the familiar case of equally-spaced observations, $t_{j+1}-$ $t_{j} \equiv 1$. Then a discrete model like (5) is obtained, with $t_{j}$ and $t_{j-1}$ replaced by $t$ and $t-1$. (Although we call it "discrete" it is of course valid continuously over $t$.) Now 
the first subvector of the latter model has the form

$$
Y(t)=\sum_{i=1}^{r} F_{i}(t) D^{i-1} Y(t-1)+\int_{t-1}^{t} G(t) d X(t)
$$

If $F_{r}(t)$ is nonsingular, an expression for $D^{r-1} Y(t-1)$ is obtained. By means of this, $D^{r-1} Y(t-1)$ is eliminated from the remainder of (5), and a new (2) is formed, in which $Y(t)$ is replaced by

$$
\left(Y(t)^{\prime}, Y(t-1)^{\prime}, D Y(t)^{\prime}, D Y(t-1)^{\prime}, \cdots, D^{r-2} Y(t)^{\prime}, D^{r-2} Y(t-1)^{\prime}\right)^{\prime}
$$

A new (5) is derived and from the first two subvectors of this, expressions for $D^{r-2} Y(t-$ 1), $D^{r-2} Y(t-2)$, deduced, much as before. A further (2) is constructed and so on, until the final (2) has $Y(t)^{\prime}$ replaced by $\left(Y(t)^{\prime}, \cdots, Y(t-r+1)^{\prime}\right)$. From the first subvector of the associated (5) model we deduce

$$
Y(t)-\sum_{j=1}^{r} R_{j}(t) Y(t-j)=\sum_{j=0}^{r-1} S_{j}(t) Z(t-j),
$$

for suitable matrices $R_{1}(t), \ldots, R_{r}(t), S_{1}(t), \ldots, S_{r-1}(t)$, and where for each $t$, the sequence $Z(t-1), Z(t), \cdots$ are uncorrelated but possibly heteroscedastic vectors, and the right side of (8) results from the $r-1$ integrations that have been performed. The fact that (7) and (8) have solutions with identical autocovariance properties may be demonstrated as in the Theorem.

Similar results were earlier established for the special case that coefficients and sampling intervals are constant over time, early references being Bartlett (1946), 
Walker (1950), Phillips (1959). Then if the initial conditions are at $-\infty$, and, say, $Y(t)$ is covariance stationary, (5) and (8) become special cases of ARMA models, about which a great deal is known. An open question is the extension of such results to spatial and spatio-temporal data.

\section{Modelling and Inference Issues}

The properties we have developed demonstrate some equivalence between continuoustime models with time-varying coefficients, and discrete-time models, but they fall far short of providing a basis for statistical inference, given discrete-time data..

The approach taken to modelling $A(t)$ and $B(t)$ in (2) is a crucial initial consideration. They could be parametric, in the sense of depending on $t$ and finitely many unknown parameters, or they could be nonparametric, or else a semiparametric setting could be considered, for example $A(t)$ could be parametric but $B(t)$ nonparametric. Then the corresponding discrete model (5) inherits the same kind of properties (though the inovations covariance matrix $\Sigma_{j}$ depends on on both $A(t)$ and $B(t)$. For parametric $A(t)$, however, the extent to which a parametric representation

of the autoregressive coefficient matrix $\Phi\left(t_{j}\right)^{-1} \Phi\left(t_{j-1}\right)$ can be deduced is far from clear. Even in the equally-spaced context, with $t_{j+1}-t_{j}$ constant, matters are more complicated even than in the time-invariant settings developed by Rex Bergstrom. Rather, a desire for efficiency gains from utilising the restrictions required by the 
continuous time model is liable to give way to a more pragmatic approach to specifying the discrete-time model, either parametrically or nonparametrically, with the continuous time one essentially playing the role of motivating a loosely-defined discrete time dynamic structure, via the Theorem. In higher-order models (7), choice of the order $r$ is as always an issue. Notice that $A(t)$ and $B(t)$ could also be regarded as stochastically generated but independent of $\{X(t)\}$ (see e.g. Nicholls and Quinn 1981), in which case the Theorem is valid conditional on $\{A(t)\},\{B(t)\}$.

Given a discrete-time specification, a variety of strategies for estimation are possible. In parametric models, these are likely to be based on a form of Gaussian likelihood or pseudo-likelihood, as in the time-invariant models considered by Rex Bergstrom. For the purpose of statistical inference, developing asymptotic distribution theory presents formidable difficulties. The irregular spacing permitted in the Theorem is a severe complication in itself, even for time-invariant models. This is due essentially to the loss of Toeplitz structure in the covariance matrix of the data, making it very difficult to develop regularity conditions that comprehensibly separate out assumptions on the process generating the observation timest, be it deterministic or stochastic, and assumptions on $\{A(t)\},\{B(t)\},\{X(t)\}$. For a vector, time-invariant version of (2), with irregular spacing, Robinson (1977) developed Gaussian estimates and asymptotic theory. In a purely discrete-time setting, Dunsmuir (1983) developed asymptotic theory for estimates of time-invariant ARMA models when data are sub- 
ject to missing from a regularly-spaced grid. For time-varying discrete-time models, asymptotic theory will rely inter alia on stability properties, or suitable knowledge of the nature of any instability. Some theoretical properties of time-varying discrete time models, with regularly-spaced observations, have been developed by, e.g., Hallin (1978), Melard and Herteleer-de-Schulter (1989), and parametric estimation has been considered by, e.g., Subba Rao (1970), Kitagawa and Gersch (1985). For nonparametric $A(t)$ and $B(t)$, a local kernel smoothing approach can be employed, as in the timevarying semiparametric regression and regularly-spaced locally stationary time series contexts of Robinson (1988), Dahlhaus (2000), respectively; here, to enable a useful asymptotic theory, $A(t)$ would be replaced by $A(t / n)$, where $n$ is the number of observations and observations are drawn at $t=1, \ldots, n$. The above discussion has had in mind the closed systems which are the focus of the paper. With open systems, briefly discussed in Section 2, after making the interpolation/approximation that creates a discrete-time model, there is potential for less dependence on strong assumptions on the input noise $X(t)$, as stressed by Robinson (1976), in case of constant-coefficient models. Rex Bergstrom's pioneering investigations have not only solved important issues in time series modelling and inference, but opened the way for much further research.

\section{Acknowledgements}

I am grateful for the helpful comments of Peter Phillips and three referees. 


\section{REFERENCES}

Bartlett, M.S. (1946) On the theoretical specification of sampling properties of autocorrelated time series. Journal of the Royal Statistical Society Supplement 8, $27-41$.

Bergstrom, A.R. (1966) Nonrecursive models as discrete approximations to systems of stochastic differential equations. Econometrica 34, 173-182.

Bergstrom, A.R. (1967) The Construction and Use of Economic Models. London: English University Press.

Bergstrom, A.R. (1976) Statistical Inference in Continuous-Time Economic Models. Amsterdam: North-Holland.

Bergstrom, A.R. (1983) Gaussian estimates and structural parameters in higherorder continuous-time dynamic models. Econometrica 51, 117-162.

Bergstrom, A.R. (1984) Monetary fiscal and exchange rate policy in a continuoustime model of the United Kingdom. In P. Malgrange and P. Muel (eds.) Contemporary Macroeconomic Modelling, Chapter 8 and pp.183-206. Oxford: Blackwell.

Bergstrom, A.R. (1988) The history of continuous-time econometric models. Econometric Theory 4, 365-383. 
Bergstrom, A.R. and Nowman, B. (2007) A Continuous Time Econometric Model of the United Kingdom with Stochastic Trends Cambridge: Cambridge University Press.

Coddington, E.A. and Levinson, N. (1955) Theory of Ordinary Differential Equations. New York: McGraw-Hill.

Dahlhaus, R. (2000) A likelihood approximation for locally stationary processes. The Annals of Statistics 28, 1762-1794.

Dunsmuir, W.T.M. (1983) A central limit theorem for estimation in Gaussian stationary time series observed at unequally spaced times. Stochastic Processes and their Applications 14, 279-295.

Friedman, A. (1975) Stochastic Differential Equations and Applications. New York: Academic Press.

Hallin, M. (1978) Mixed autoregressive-moving average multivariate processes with time-dependent coefficients. Journal of Multivariate Analysis 8, 567-572.

Kitagawa, G. and Gersch, W. (1985) A smoothness priors time-varying AR coefficient modeling of nonstationary covariance time series. IEEE Transactions on Automatic Control AC-30, 48-55. 
Melard, G. and Herteleer-de-Schutter, A. (1989) Contributions to evolutionary spectral theory. Journal of Time Series Analysis 10, 41-63.

Nicholls, D. and Quinn, B.G. (1980) The estimation of random coefficient autoregressive models I. Journal of Time Series Analysis 1, 37-46.

Phillips, A.W. (1959) The estimation of parameters in systems of stochastic differential equations. Biometrika 46, 67-76.

Phillips, P.C.B. (1973) The problem of identification in finite parameter continuous time models. Journal of Econometrics 1, 351-362.

Phillips, P.C.B. (1974) The estimation of some continuous time models. Econometrica $42,803-823$.

Phillips, P.C.B. and Yu, J. (2005a) Jackknifing bond option prices. Review of Financial Studies 18, 707-742.

Phillips, P.C.B. and Yu, J. (2005b) Comments: A selective overview of nonparametric methods in financial econometrics. Statistical Science 20, 338-343.

Phillips, P.C.B. and J. Yu (2006) Maximum likelihood and Gaussian estimation of continuous time models in finance. Working paper, Coweles Founation for Research in Economics. 
Robinson, P.M. (1976) The estimation of linear differential equations with constant coefficients. Econometrica 44, 751-764.

Robinson, P.M. (1977a) The construction and estimation of continuous time models and discrete approximations in econometrics. Journal of Econometrics 6, 173197.

Robinson, P.M. (1977b) Estimation of a time series model from unequally spaced data. Stochastic Processes and their Applications 6, 9-24.

Robinson, P.M. (1989) Nonparametric estimation of time-varying parameters. Statistical Analysis and Forecasting of Economic Structural Change (P. Hackl, ed.), Springer-Verlag, 253-264.

Sargan, J.D. (1974) Some discrete approximations to continuous time stochastic models. Journal of the Royal Statistical Society 36, 74-90.

Soong, T.T. (1973) Random Differential Equations in Science and Engineering. New York: Academic Press.

Subba Rao, T. (1970) The fitting of non-stationary time-series models with timedependent parameters. Journal of the Royal Society B, 312-322.

Walker, A.M. (1950) Note on a generalization of the large sample goodness of fit test for linear autoregressive schemes. Journal of the Royal Statistical Society 
B12, 102-107. 